\title{
Unveiling modal parameters with forced response using SVD and QR during Flutter Flight Testing
}

\author{
José Barros-Rodríguez ${ }^{1}$, José Miguel Fernández Fructuoso ${ }^{2}$, Roberto Flores Le Roux ${ }^{3}$, Sebastian Sanchez Prieto ${ }^{4}$ \\ and Oscar Rodríguez Polo ${ }^{5}$
}

\begin{abstract}
This paper presents a method for identification of modal parameters during flutter flight testing when excitation is employed and the aircraft possesses several acquisition sensors. The method takes advantage of the capacity of SVD for the analysis and identification of matrix rank, the power of QR factorization to reorder the files in a matrix in accordance with the information provided, general vector field properties as well as the features of exponentially-damped harmonic functions and the properties of the response resulting from the convolution of this type of functions with an excitation signal. The method is a generalization of an algorithm used at CLAEX (Spanish Flight Test Centre) to identify the free response of the aircraft structure after fix frequency excitation during in-flight flutter testing. Application to analytic signals as well as structural response of modern fighter aircraft using frequency sweep excitation is provided in order to demonstrate the effectiveness, robustness and noise immunity of the proposed method.
\end{abstract}

Keywords: flight flutter; damping; modal parameters identification; SVD, QR factorization

\section{Background}

During Flutter Flight Testing, contrary to Ground Vibration Tests, the short duration of the signals and the closeness of the frequencies as well as difference in energy of such signals, make modal identification of critical modes (those responsible of flutter phenomena) particularly difficult.

Two types of excitations are frequently employed in modern fighter Flutter Flight Testing: Sweep Frequency aileron deflection excitation that is used to identify structural modal parameters and Dwell (few seconds fixed frequency) aileron deflection excitation that is used to obtain an estimation of damping and to identify non-linear effects.

The Singular Value Decomposition (SVD) and Column Pivoting Method works in the time domain and has been employed successfully after Dwell excitation to analyze the short free response of close modes.

The basic methodology has been extended to enable modal analysis of the forced response.

The proposed method operates in the time domain, but contrary to some tradicional time domain fitting algorithms as the Least Square Complex Exponential, or the Ibrahim Time Domain, does not make use of the impulse response function ${ }^{3}$ and so does not need to employ the Inverse Fourier transform of the FRF (Frequency Response Function), since the proposed method uses directly excitation and response signals. A time domain method using directly excitation and response signals is ERA (Eigenvalue Realization Algorithm) that has been developed by Juang and Pappa ${ }^{12}$ and employed by Brennen for the analysis of flutter data ${ }^{13}$.

${ }^{1}$ Senior Enginneer, jose.barrosrodriguez@occar.int, OCCAR (Organisation conjointe de coopération en matière d'armement), Seville, Spain.

${ }^{2}$ Senior Engineer, fructuoso@ea.mde.es, CLAEX (Logistic Center of Armament and Flight Test), Spanish Air Force (SAF), Madrid, Spain.

${ }^{3}$ Senior Researcher \& Professor, rflores@ cimne.upc.edu, CIMNE (Centro Internacional de Métodos Numéricos en Ingeniería) \& UPC (Universidad Politécnica de Cataluña), Barcelona, Spain.

${ }_{5}^{4}$ Senior Researcher \& Professor, sebastian.sanchez@uah.es, UAH (Universidad de Alcalá), Madrid, Spain.

${ }^{5}$ Senior Researcher \& Professor, o.rodriguez@uah.es, UAH (Universidad de Alcalá), Madrid, Spain.

Journal of Aerospace Engineering

http://mc.manuscriptcentral.com/(site) 
These mentioned methods are very sensitive to the presence of noise and non-linearities. In order to overcome this problem, several new methodologies have been developed. The HHT (Hilbert-Huang Transform), employed by Brennen and Prazenica for the analysis of flutter data ${ }^{10}$ as well as by Islam Moya for the analysis of Climate Variability ${ }^{11}$, decomposes the signal in intrinsic mode functions by means of EMD (Empirical Mode Decomposition) and applies the Hilbert Transformation to each one of these functions. Alternatively, time-frequency domain, in particular Waveltet filtering, can be employed in the cases of sweep excitation to try to eliminate the contributions of non-linear phenomena ${ }^{7-9}$. The Wavelet Transformation, specifically the Morlet Wavelet, is employed by Kijewski and Kareem ${ }^{2}$ as well as by Slavic and Boltezar ${ }^{4-6}$ for system identification. The method proposed is able in some cases to surmount noise and non-linearities by looking in a interval of time where these problems are negligible.

\section{Principles of the Method}

The method is based on several principles and hypothesis being linearity of the system the basic one. As a direct consequence the structural modes are represented by exponentially-damped harmonic functions.

\section{Hypotesis of linearity}

The basic hypothesis used in this method is that there is a structural equivalent system in which the structural modes can be represented by harmonic signals exponentially damped ${ }^{1-3}$. This assumption implies the existence of a standard set of linearized equations of motion:

$$
M \ddot{h}+C \dot{h}+K h=0
$$

where $\mathrm{M}, \mathrm{C}$ and $\mathrm{K}$ denote the mass, damping and stiffness matrices respectively and $\mathrm{h}(\mathrm{t})$ is the displacement vector. Since in this case the equation is homogeneous without excitation, $h(t)$ is the impulse response or the free response of the system. The solution of Eq. (1) can be expressed as a function of the modal coordinates as:

$$
h(t)=\Phi q(t)=\sum_{r=1}^{N} \varphi^{r} q^{r}(t)
$$

In equation (2) $\Phi$ denotes the modal matrix whose rows are the modal shape vectors $\varphi^{\mathrm{r}}$. This matrix depends on the initial conditions of the system. The vector $q(\mathrm{t})$ contains the values of the modal coordinates $\mathrm{q}^{\mathrm{r}}(\mathrm{t})$. The time evolution of the modal coordinates is given by:

$$
q^{r}(t)=e^{-\xi^{r} 2 \pi n_{n}^{r} t} \sin \left(2 \pi f_{d}^{r} t+\phi^{r}\right) \quad f_{d}^{r}=f_{n}^{r} \sqrt{1-\xi^{r 2}}
$$

$f_{d}^{r}$ denotes the damped frequency of mode $\mathrm{r}$, whose natural frequency and damping ratio are $f_{n}^{r}$ and $\xi^{\mathrm{r}}$; on its side, $\varnothing^{r}$ is the phase of the mode $r$.

Property of exponentially damped harmonic signals ${ }^{1}$

If in an exponentially damped sinusoidal signal, three portions corresponding to three intervals of time of the same length are taken, then the vectors describing these three portions are linearly dependent, unless two of them have the same phase. This means any of these three vectors can be built as linear combination of the other two. In case the signal is formed by two exponentially damped sinusoidal signals, then four portions corresponding to four intervals of time have to be taken to build any other portion of the signal and so on. 
Response of the system to excitation

When the system is submitted to an exciting force:

$$
M \ddot{x}+C \dot{x}+K x=f(t)
$$

And assuming that $\mathrm{h}(\mathrm{t})$ is the solution of equation $(1)$, the system response $\mathrm{x}(\mathrm{t})$ is:

$$
x(t)=\int_{0}^{t} f(\tau) \cdot h(t-\tau) \cdot d \tau
$$

It has to be taken into account that the signal from a sensor of the aircraft contains electrical noise, forced response to turbulence and the effect of nonlinearities. If the noise and response to turbulence are random and Gaussian distributed and only weak nonlinear effects are present, the method proposed hereafter is applicable.

\section{Superposition principle}

The superposition principle states that for a linear system, the net response at a given time caused by two or more excitation forces is the sum of the responses which would have been caused by each force individually. This means that equation (4) can be written as:

$$
\begin{array}{llll}
M \ddot{x}_{1}+C \dot{x}_{1}+K x_{1}=f(t) & \text { if } t<T ; & M \ddot{x}_{1}+C \dot{x}_{1}+K x_{1}=0 & \text { if } t \geq T \\
M \ddot{x}_{2}+C \dot{x}_{2}+K x_{2}=0 \quad \text { if } t<T ; & M \ddot{x}_{2}+C \dot{x}_{2}+K x_{2}=f(t) & \text { if } t \geq T \\
x(t)=x_{1}(t)+x_{2}(t) \quad \forall T & &
\end{array}
$$

Taking into account the superposition principle, the Eq. (5) can be rewritten as:

$$
x(t)=\int_{T}^{t} f(\tau) \cdot h(t-\tau) \cdot d \tau+\tilde{h}(t) ; \quad \tilde{h}(t)=\widetilde{\Phi} q(t)=\sum_{r=1}^{N} \tilde{\varphi}^{r} q^{r}(t)
$$

In equation (7) $\tilde{\Phi}$ denotes the modal matrix whose rows are the modal shape vectors $\tilde{\varphi}^{r}$. This matrix depends on the initial conditions of the system at the moment $T$.

The response of the system after the moment $T$ is a composition of the response due to an excitation $f(t)$ beginning at $\mathrm{T}$ and the free response of the system.

\section{Multiple sensors}

Flutter flight testing is performed using a test aircraft with several accelerometers and strain gauges embedded in the structure and in the loads (external fuel tanks, bombs, pods, missiles, etc) corresponding to the configuration under test. Since the aircraft structure is excited by two signals moving both ailerons, either in phase or in 180 degrees out-of-phase phase, in order to detect symmetric and antisymmetric modes respectively, it can be modeled by a single input system.

Each modal shape vector $\varphi^{\mathrm{r}}$ of the modal matrix $\Phi$ in equation (2) represent the amplitudes of the modes detected by a sensor. Every sensor detects each mode with an amplitude that depends on its location. If, however, the sensor is placed close to a node, the corresponding mode cannot be detected because the signal it creates is too weak (i.e. the relevant component of the modal vector is small) .

Considering that the aircraft has $\mathrm{P}$ sensors and $\mathrm{N}$ structural modes, that the impulse response of sensor $\mathrm{i}$ is $h_{i}(t)$, and that its response $x_{i}$ that begins at $t=0$ and is sampled and evaluated between $t=T_{1}$ and $t=T_{n}$, then:

$$
x_{i}=\left[x_{i}\left(T_{1}\right) x_{i}\left(T_{2}\right) \cdots x_{i}\left(T_{n}\right)\right]=h_{i} * f=\left[h_{i}\left(T_{1}\right) h_{i}\left(T_{2}\right) \cdots h_{i}\left(T_{n}\right)\right] *\left[f\left(T_{1}\right) f\left(T_{2}\right) \cdots f\left(T_{n}\right)\right]
$$

And representing the impulse response by its modes: 


$$
\begin{aligned}
\begin{array}{l}
x_{1}= \\
\vdots
\end{array} & \varphi_{1}^{1}\left[f * q^{1}\right]+\cdots+\varphi_{1}^{r}\left[f * q^{r}\right]+\cdots+\varphi_{i}^{N}\left[f * q^{N}\right] \\
\vdots & \\
x_{i}=f * \sum_{1}^{N} \varphi_{i}^{r} q^{r}=\sum_{1}^{N} \varphi_{i}^{r}\left[f * q^{r}\right] & =\varphi_{i}^{1}\left[f * q^{1}\right]+\cdots+\varphi_{i}^{r}\left[f * q^{r}\right]+\cdots+\varphi_{i}^{N}\left[f * q^{N}\right] \\
\vdots & \\
x_{P}= & =\varphi_{P}^{1}\left[f * q^{1}\right]+\cdots+\varphi_{P}^{r}\left[f * q^{r}\right]+\cdots+\varphi_{P}^{N}\left[f * q^{N}\right]
\end{aligned}
$$

It can be noticed that in this equation system the left side is known and the right side is unknown. In the right side there are PxN unknown parameters $\varphi_{i}^{r}$ and $\mathrm{N}$ unknown $\left[f * q^{r}\right]$ vectors. This means that taking $\mathrm{N}$ vectors in the left side $\mathrm{xi}$ and in case they are not linearly dependent, it is possible to form a base equivalent to the $\left[f * q^{r}\right]$ vectors.

\section{Proposed method for system identification}

\section{Number of modes}

In order to be able to use the method the number of sensors available must be equal or higer than the number of modes, that means $\mathrm{P}>\mathrm{N}$. Even when the aircraft has infinite modes of vibration, in most of the cases the method is valid since only a few modes are excited enough to be detected and relevant in the analysis.

Let us take the response signals between $\mathrm{T}_{1}$ and $\mathrm{T}_{\mathrm{n}}$ and recorded with sampling frequency fs:

$$
x_{i}=\left[x_{i}\left(T_{1}\right) x_{i}\left(T_{2}\right) \cdots x_{i}\left(T_{n}\right)\right] \quad i=1 \cdots P
$$

The first step of the method is to build a matrix A with the response signals:

$$
A=\left[\begin{array}{cccc}
x_{1}\left(T_{1}\right) & x_{1}\left(T_{2}\right) & \cdots & x_{1}\left(T_{n}\right) \\
x_{2}\left(T_{1}\right) & x_{2}\left(T_{2}\right) & \cdots & x_{2}\left(T_{n}\right) \\
& & \vdots & \\
x_{P}\left(T_{1}\right) & x_{P}\left(T_{2}\right) & \cdots & x_{P}\left(T_{n}\right)
\end{array}\right]
$$

In light of equation (9), for a linear and free of noise, the rank of matrix A (the number of nonzero singular values on its SVD) should match the number of modes. On the other hand, in a real system with noise, turbulence and weak nonlinearity all the singular values are nonzero. The smallest singular values appear due to these additional factors.

$$
A=U S V^{T} \Rightarrow S=\overbrace{\left[\begin{array}{cccccc}
s_{1} & 0 & \cdots & 0 & \cdots & 0 \\
0 & s_{2} & \cdots & 0 & \cdots & 0 \\
& & \ddots & & & \\
0 & 0 & \cdots & s_{P} & \cdots & 0
\end{array}\right]}^{n} \Rightarrow s_{1} \geq s_{2} \geq \cdots \geq s_{P} \geq 0
$$


If the conditions under which the method is applicable are met, there will be $\mathrm{N}$ dominant singular values with the rest being considerably smaller. This provides a direct measure of the modal order of the system. If there is no clear distinction between both sets of singular values this means the method is not applicable (e.g. there are strong nonlinearities in the response). By dropping the small singular values it is possible to filter out most of the noise. This is achieved by building the matrix $A_{C}$ which is almost noise-free, in which small singular values have being equalled to zero:

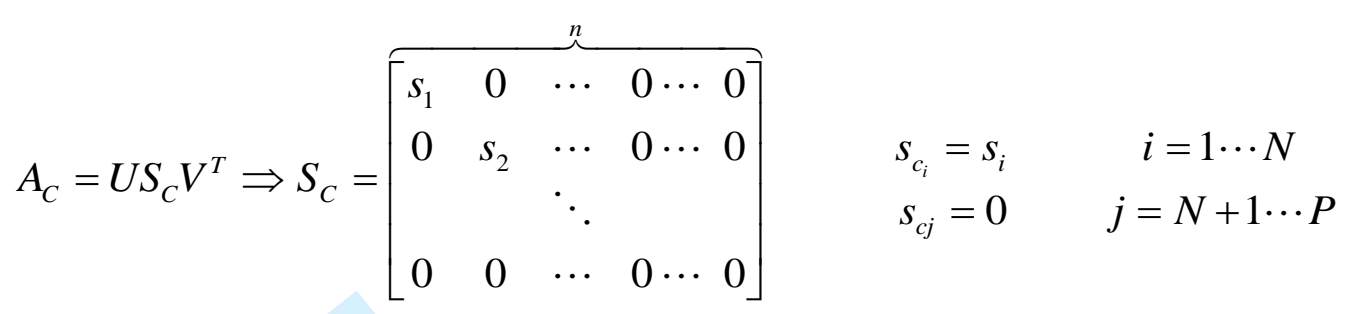

QR factorization and file pivoting

Any set of $\mathrm{N}$ files from matrix $\mathrm{A}_{\mathrm{C}}$ would form a base equivalent to the $\mathrm{N}$ vectors $\left[f * q^{r}\right]$. It is important, however, to choose the most adequate vectors. These are the vectors that are the most orthogonal to each other and have maximum norm. An efficient means of achieving this goal is $\mathrm{QR}^{[12,15]}$ factorization with file pivoting using the permutation matrix E such that:

$$
A_{C}^{T} E=Q R \quad / \quad Q Q^{T}=I \quad, \quad r_{i j}=0 \forall i>j \quad \Leftrightarrow \quad B=E^{T} A_{C}
$$

The matrix $\mathrm{B}$ is ordered in such a way that its first $\mathrm{N}$ rows are the best base available to represent the space spanned by the vectors vectors $\left[f * q^{r}\right]$. Therefore, it is enough to keep these $\mathrm{N}$ rows and the rest can be discarded. A truncated matrix $\mathrm{B}_{\mathrm{t}}$ can be built keeping only the first $\mathrm{N}$ rows:

$$
B_{t}=\left[\begin{array}{cccc}
x_{\tau}\left(T_{1}\right) & x_{\tau}\left(T_{2}\right) & \cdots & x_{\tau}\left(T_{n}\right) \\
x_{\pi}\left(T_{1}\right) & x_{\pi}\left(T_{2}\right) & \cdots & x_{\pi}\left(T_{n}\right) \\
& & \vdots & \\
x_{\psi}\left(T_{1}\right) & x_{\psi}\left(T_{2}\right) & \cdots & x_{\psi}\left(T_{n}\right)
\end{array}\right] \quad N \text { rows }
$$

Since matrix $B_{t}$ is of rank $N$, its SVD has a singular matrix $S$ (N by $n$ ) with $N$ nonzero singular values. Column pivoting ${ }^{[6]}$ based on QR decomposition in order to optimize row selection in matrix $\mathrm{A}$ is not employed in other modal analysis methods and is inspired by Prof. Kanjilal ${ }^{[6]}$ subset selection.

Frequency and damping determination

An augmented matrix $\mathrm{B}^{*}$ can be obtained adding an additional row to Bt. If the new row is linearly independent from the old ones (i.e. it is linearly independent from the $\mathrm{N}$ vectors $\left[f * q^{r}\right]$ or equivalently from the $\mathrm{N}$ vectors $\mathrm{x}_{\mathrm{i}}$ for $\mathrm{i}=\tau, \pi, \ldots \psi$ ) $\mathrm{B}^{*}$ will be of rank $2 \mathrm{n}+1$. On the other hand, if the new vector is a linear combination of the rows of $\mathrm{B}_{t}$, the ranks of $\mathrm{B}_{\mathrm{t}}$ and $\mathrm{B}^{*}$ shall remain equal due to the additional singular value being null.

A sensor able to detect a single mode would measure the response of such structural mode to the excitation and could provide a vector that would be linearly dependent of the files of matrix $\mathrm{B}_{\mathrm{t}}$.

In order to find the modes contained in the signal a parametric family of exponentially-damped sinusoidal functions (normalized with their Euclidean norm) is created. The free parameters are the frequency and 
the reduced damping $\left(f_{j}, \xi_{\mathrm{j}}\right)$. An additional parameter would be the phase, but in order to avoid it, two functions in 90 degrees out of phase are generated.

$$
\begin{aligned}
& y_{j}^{C}(t)=e^{-\xi_{j} 2 \pi f_{j} t} \cos \left(2 \pi f_{j} t\right) \Rightarrow Y_{j}^{C}=\left[y_{j}^{C}\left(T_{1}\right) y_{j}^{C}\left(T_{2}\right) \cdots y_{j}^{C}\left(T_{n}\right)\right] \Rightarrow Y_{j}^{C 1}=\frac{Y_{j}^{C}}{\left\|Y_{j}^{C}\right\|} \\
& y_{j}^{S}(t)=e^{-\xi_{j} 2 \pi f_{j} t} \sin \left(2 \pi f_{j} t\right) \Rightarrow Y_{j}^{S}=\left[y_{j}^{S}\left(T_{1}\right) y_{j}^{S}\left(T_{2}\right) \cdots y_{j}^{S}\left(T_{n}\right)\right] \Rightarrow Y_{j}^{S 1}=\frac{Y_{j}^{S}}{\left\|Y_{j}^{S}\right\|}
\end{aligned}
$$

Evaluating the convolution of these two functions with the excitation force considering that such a force is equal to zero outside the period between $\mathrm{t}=\mathrm{T}_{1}$ and $\mathrm{t}=\mathrm{T}_{\mathrm{n}}$, then:

$$
\begin{aligned}
& R_{j}^{C}=Y_{j}^{C 1} * f=\left[y_{j}^{C 1}\left(T_{1}\right) y_{j}^{C 1}\left(T_{2}\right) \cdots y_{j}^{C 1}\left(T_{n}\right)\right] *\left[f\left(T_{1}\right) f\left(T_{2}\right) \cdots f\left(T_{n}\right)\right] \\
& R_{j}^{S}=Y_{j}^{S 1} * f=\left[y_{j}^{S 1}\left(T_{1}\right) y_{j}^{S 1}\left(T_{2}\right) \cdots y_{j}^{S 1}\left(T_{n}\right)\right] *\left[f\left(T_{1}\right) f\left(T_{2}\right) \cdots f\left(T_{n}\right)\right]
\end{aligned}
$$

In case the parameters of frequency and reduced damping correspond to one structural mode, then due to the superposition principle the response of the system due to this mode to the excitation force is a combination of the four vectors $Y_{j}^{C 1}, Y_{j}^{S 1}, R_{j}^{C}, R_{j}^{S}$.

The $Y_{j}^{C 1}, Y_{j}^{S 1}, R_{j}^{C}, R_{j}^{S}$ vectors are used to build a family of augmented matrices $B_{j}^{*}$. The SVD transformation is then applied to each member of this family:

$$
f_{j} \rightarrow Y_{j}^{Y_{j}^{C 1}} \rightarrow{ }_{R_{j}^{S}}^{R^{C}} \rightarrow B_{j}^{*}=\left[\begin{array}{cccc}
x_{\tau}\left(T_{1}\right) & x_{\tau}\left(T_{2}\right) & \cdots & x_{\tau}\left(T_{n}\right) \\
x_{\pi}\left(T_{1}\right) & x_{\pi}\left(T_{2}\right) & \cdots & x_{\pi}\left(T_{n}\right) \\
& & \vdots & \\
x_{\psi}\left(T_{1}\right) & x_{\psi}\left(T_{2}\right) & \cdots & x_{\psi}\left(T_{n}\right) \\
y_{j}^{C 1}\left(T_{1}\right) & y_{j}^{C 1}\left(T_{2}\right) & \cdots & y_{j}^{C 1}\left(T_{n}\right) \\
y_{j}^{S 1}\left(T_{1}\right) & y_{j}^{S 1}\left(T_{2}\right) & \cdots & y_{j}^{S 1}\left(T_{n}\right) \\
r_{j}^{C}\left(T_{1}\right) & r_{j}^{C 1}\left(T_{2}\right) & \cdots & r_{j}^{C 1}\left(T_{n}\right) \\
r_{j}^{S}\left(T_{1}\right) & r_{j}^{S}\left(T_{2}\right) & \cdots & r_{j}^{S}\left(T_{n}\right)
\end{array}\right] \quad N+4 \text { rows } \stackrel{S V D}{\longrightarrow} B_{j}^{*}=U_{j} S_{j} V_{j}^{T}
$$

To evaluate the rank of $B_{j}^{*}$ the product of the N+4 singular values of $\mathrm{S}_{\mathrm{j}}$ is taken. Let us denote it by $\Psi_{\mathrm{j}}$.

$$
S_{j}=\left[\begin{array}{cccccc}
s_{j}^{1} & 0 & \cdots & 0 & \cdots & 0 \\
0 & s_{j}^{2} & \cdots & 0 & \cdots & 0 \\
\vdots & & \ddots & \vdots & & \vdots \\
0 & 0 & \cdots & s_{j}^{N+4} & \cdots & 0
\end{array}\right] \Rightarrow \Psi_{j}=s_{j}^{1} \cdot s_{j}^{2} \cdots s_{j}^{N+4}
$$

Note that $\Psi_{\mathrm{K}}$ is actually the determinant of the reduced matrix obtained retaining only the first N+4 columns of $\mathrm{S}_{\mathrm{j}}$. This determinant vanishes if matrix $B_{j}^{*}$ is of rank $\mathrm{N}+3$. For each pair of parameters $\left(\mathrm{f}_{\mathrm{j}}, \xi_{\mathrm{j}}\right)$ a $\Psi_{\mathrm{j}}$ value can be computed which assesses to what degree the combination of the four vectors $Y_{j}^{C 1}, Y_{j}^{S 1}, R_{j}^{C}, R_{j}^{S}$ is independent from the $\left[f * q^{r}\right]$ base. Those combinations which more closely match the modes of the system yield minimum values of $\Psi_{\mathrm{j}}$. 
In order to obtain a comprehensive coverage a sequence of $\left(f_{j}, \xi_{j}\right)$ pairs must be built which spans the range of interest with an adequate resolution. For each frequency different values of the damping ratio are tested in order to determine the minimum $\Psi$ for a given frequency. Then, the process is repeated for all the remaining values of the frequency. The local minimums of the $\Psi$ function indicate the $\left(f_{j}, \xi_{\mathrm{j}}\right)$ pairs which more closely approximate the modal parameters (frequency and damping) of the structure.

\section{Robustness of the Results}

While SVD is a very robust algorithm for matrix analysis it cannot always avoid spurious matches between the $Y_{j}^{C 1}, Y_{j}^{S 1}, R_{j}^{C}, R_{j}^{S}$ vectors and noise present in the signal. Nevertheless, it must be noted that the analytical vectors were built using the sine and cosine functions, but could have been built from arbitraries phases between the functions. In case a real mode has been identified the values of $\Psi_{\mathrm{j}}$ for vectors built with both sine and cosine functions or any arbitrary phase must reach a local minimum. Failure to this condition indicates that the mode identifies is spurious and must be discarded.

The method proposed is not overly sensitive to the order of the system (the number of modes retained N) contrary to other techniques of Modal Analysis. Experiments with real and analytical signals have demonstrated that the results do not change appreciably when the number of rows retained in matrix $\mathrm{Bt}$ is larger than the number of modes.

\section{Results and discussion}

Tests were conducted using the basic MATLAB 6.5 software without specialized toolboxes, applying the method to both synthetic data and real flight data.

Application to synthetic data

The synthetic data has been built up with a sweep frequency excitation signal $\mathbf{f}$ and ten responses $\mathbf{x}^{\mathbf{i}}$ that where produced by convolution of this excitation signal with four impulse responses that represents four structural modes $\mathbf{q}^{\mathbf{i}}$.

$$
\begin{aligned}
& \text { Excitation Signal } \\
& f=\left(\frac{t}{37}+1\right) \sin \left(2 \pi\left(\frac{11}{37} t+2\right) t\right)+\varepsilon(0,0.5) \\
& x_{1}=\quad 0.2\left[f * q^{2}\right]+0.5\left[f * q^{3}\right] \\
& x_{2}=\quad 0.2\left[f * q^{3}\right] \\
& \text { Im pulse Re sponse } \\
& q^{1}(f=3.1 H z ; \xi=0.072)=e^{-2 \pi f \xi t} \sin (2 \pi t) \\
& q^{2}(f=4.78 H z ; \xi=0.08) \\
& q^{3}(f=6.16 H z ; \xi=0.058) \\
& q^{4}(f=7.12 \mathrm{~Hz} \xi=0.012) \\
& x_{3}= \\
& 0.3\left[f * q^{2}\right]+0.7\left[f * q^{3}\right]-0.1\left[f * q^{4}\right]+\varepsilon_{N}(0,0.5) \\
& x_{4}=-0.3\left[f * q^{1}\right]+0.7\left[f * q^{2}\right]+0.2\left[f * q^{3}\right]+0.1\left[f * q^{4}\right]+\varepsilon_{N}(0,0.5) \\
& x_{5}=\quad-0.5\left[f * q^{3}\right]+0.5\left[f * q^{4}\right]+\varepsilon_{N}(0,0.5) \\
& x_{6}=0.3\left[f * q^{1}\right]+0.6\left[f * q^{2}\right]+0.1\left[f * q^{4}\right]+\varepsilon_{N}(0,0.5) \\
& x_{7}=0.9\left[f * q^{1}\right]-0.2\left[f * q^{2}\right]+0.3\left[f * q^{3}\right]-0.1\left[f * q^{4}\right]+\varepsilon_{N}(0,0.5) \\
& x_{8}=0.3\left[f * q^{1}\right] \quad+0.7\left[f * q^{4}\right]+\varepsilon_{N}(0,0.5) \\
& \text { Time and Sampling Frequency } \\
& t=0 \text { to } 34 \mathrm{sec} \text {. } \\
& f_{s}=100 \mathrm{~Hz} \\
& \begin{array}{l}
x_{9}=0.4\left[f * q^{2}\right]-0.2\left[f * q^{3}\right]-0.8\left[f * q^{4}\right]+\varepsilon_{N}(0,0.5) \\
x_{10}=-0.5\left[f * q^{1}\right] \\
+0.6\left[f * q^{3}\right]-0.1\left[f * q^{4}\right]+\varepsilon_{N}(0,0.5)
\end{array}
\end{aligned}
$$

Gaussian noise was superimposed with the excitation signal in order to simulate the noise in the aircraft ailerons and the result was convolved with impulse signals. In addition Gaussian noise has been added as well to the response signal in order to simulate the noise in the sensors and in the telemetry signal.

In a real flutter flight testing the frequencies are relatively well known, or at least the surroundings of such frequencies, since vibration tests on ground as well as analysis variation of the modal frequencies with speed have been performed before the flight. The main task is to identify the final frequency and most important the damping indicating the remaining structural stability. In order to find the frequency around $3 \mathrm{~Hz}$, the method is applied the signal between the instant $\mathrm{t}=0.5 \mathrm{sec}$. and $\mathrm{t}=3.5 \mathrm{sec}$. 

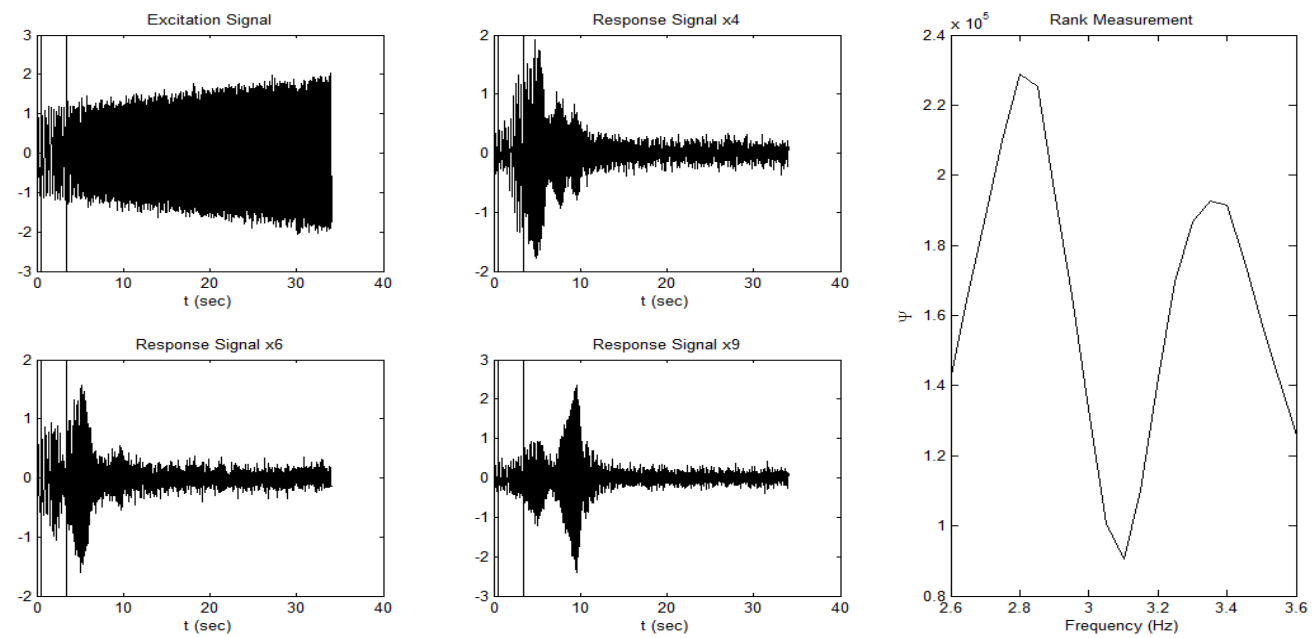

Figure 1- Method application to Synthetic Data Signals

For each frequency investigated, there is a damping value that gives a minimum for $\Psi$ parameters, that is a minimum for rank measurement.

\begin{tabular}{|l|c|c|c|c|c|c|c|c|c|c|c|}
\hline Frequency $(\mathrm{Hz})$ & 2.6 & 2.7 & 2.8 & 2.9 & 3 & 3.1 & 3.2 & 3.3 & 3.4 & 3.5 & 3.6 \\
\hline Damping & 0.01 & 0.01 & 0.01 & 0.095 & 0.08 & 0.07 & 0.075 & 0.085 & 0.01 & 0.01 & 0.01 \\
\hline$\Psi(1 \mathrm{e} 5)$ & 1.4295 & 1.8856 & 2.2878 & 1.9553 & 1.3314 & 0.90529 & 1.423 & 1.8661 & 1.9142 & 1.5856 & 1.2599 \\
\hline
\end{tabular}

Table 1- Numerical Results of Method application to Synthetic Data Signals

\section{Application to flight data}

The method has been applied to real signals from sensors embedded on the wing of the modern fighter aircraft during an representative SRAAM missile integration flutter test-flight. The computing time (around 2 to 3 second) with MATLAB software can be considered for this type of testing real time and indicates that the method could be working combined with prediction algorithms (Flutter Margin) to assist to the flight test engineer in the decision on whether to proceed to a more critical point during inflight testing.

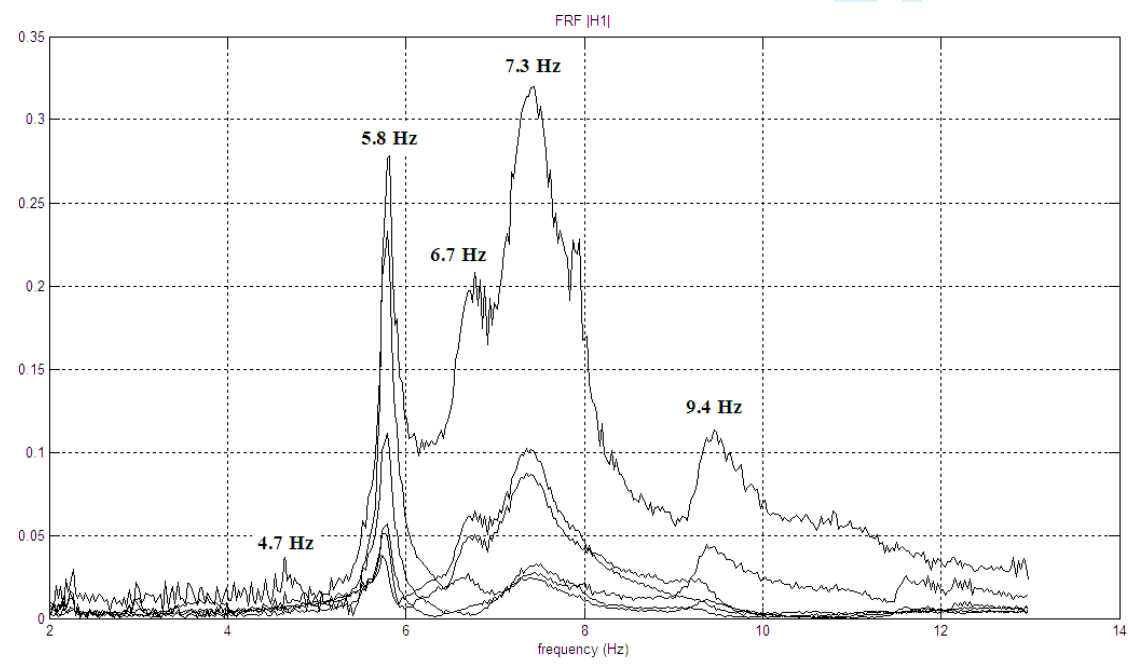

Figure 2- Frequency Response Function of modern fighter Sensors

8

Journal of Aerospace Engineering

http://mc.manuscriptcentral.com/(site) 
The method identifies the damping value of the modes corresponding to the frequency $5.8 \mathrm{~Hz}$ and frequency $7.4 \mathrm{~Hz}$ with values of 0.01 and 0.045 respctively when applied in a quite broad set of instants. It can be observed in the figure 2 that these two modes correspond to those with the higest energy. However the it is not able to identify undoubtedly the damping value of the mode corresponding to a frequency of $6.8 \mathrm{~Hz}$, that wanders between 0.03 and 0.055 . Additionally in order to identify the mode the instant had to be precisely determined in a very narrow interval of time. This mode of $6.7 \mathrm{~Hz}$ is shadowed by the $7.3 \mathrm{~Hz}$ mode since they are very close and the latter one has much more energy. Finally the method fails to identify the last mode corresponding to a frequency of $9.4 \mathrm{~Hz}$. The results of the analysis are summarized in figure 3 .
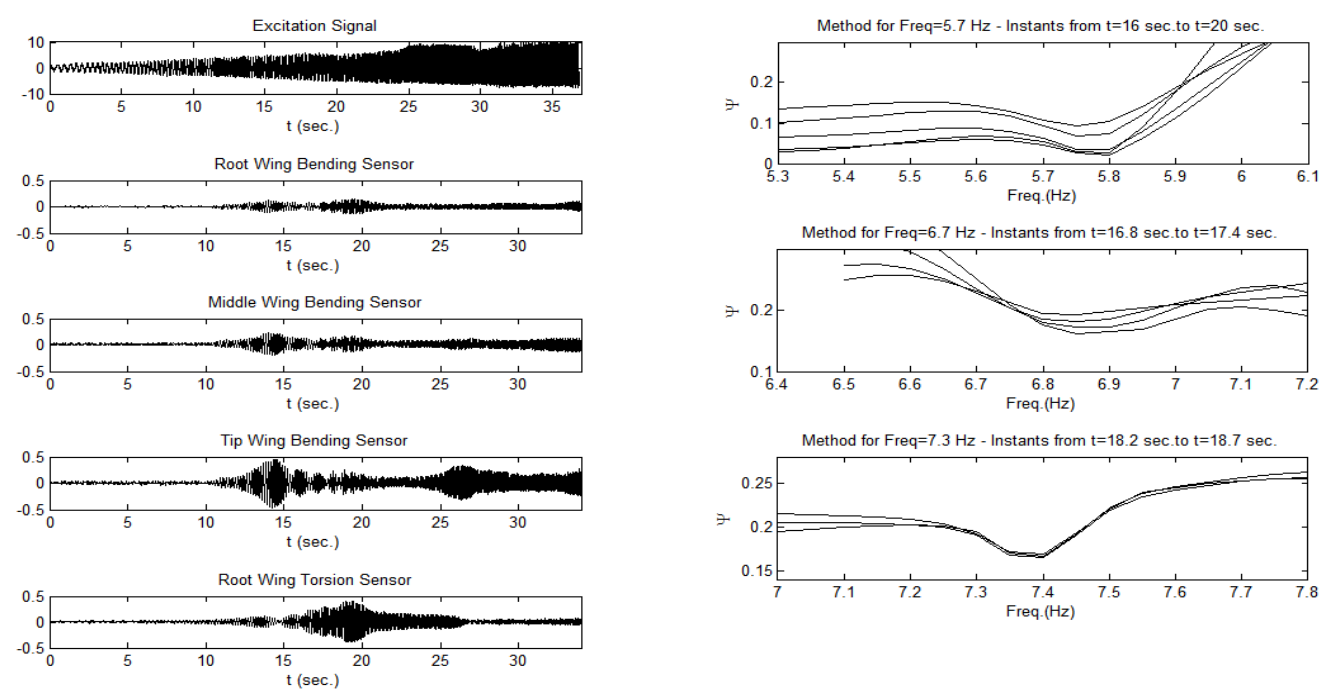

Figure 3- Modern fighter Sensors and method application

\section{Conclusions}

A method based in SVD, QR factorization, vector field properties and features of exponentially-damped harmonic functions, considering a lineal system has been presented when excitation is employed and several acquisition sensors are available in the aircraft. The method uses only segments of time to identify the modal parameters instead of using the complete period of excitation. The method remains in the domain of time and does not need to identify the impulse response of the system as most of the modal identification methods ${ }^{3}$. Time-shifting of the response signal yields a symmetric square matrix from which, the modal order of the system is determined. Next, using QR factorization, a base of the space spanned by the modal vectors is built. Finally, SVD is utilized for modal parameters identification using a family of sinusoidal exponentially-damped functions.

The method overcomes the signal identification limitations found with testing flight conditions near flutter, due to the proximity of adjacent modes and the short sample length. The proposed method successfully deals as well the so frequent short time signal loss since not the complete signal is required.

The method described was applied to an anlystic example as well as to flutter flight-test data gathered during the SRAAM / modern fighter integration campaign. The technique demonstrated adequate modal identification performance (in terms of damping and frequency).

Further studies to evaluate the impact in accuracy of non-linearity, sampling frequency, signal length, noise content and relative amplitude and damping of neighbouring modes as well as the development of an error-estimation criteria are underway. Additional work will be devoted in order to reduce the current time using tools as Simulink Coder in order to mígrate the Matlab algorithm to C Coding. 


\section{References}

1. Frequency and damping identification in flutter flight testing using Singular Value Decomposition (SVD) and QR factorization - J. Barros, R. Flores Le Roux, J López-Díez and R. Martinez-Val - Journal of Aeroespace Engineering, Sagepub UK - Accepted for publishing 04-Apr-2014.

2. Wavelelet Transforms for System Identification in Civil Engineering - Tracy L. Kijewski, Ahsan Kareem - 2003

3. Modal Analysis - Jimin He \& Zhi-Fang Fu - Butterworth Heinemann, First Edition 2001

4. Damping Identification with the Morlet-Wave - Janko Slavic, Miha Boltezar - 2011

5. Damping identification using a continuous wavelet transform: application to real data - Janko Slavic, Miha Boltezar - Journal of Sound and Vibration 262 (2003).

6. Enhancements to the continuous wavelet transform for damping identifications on short signals - Janko Slavic, Miha Boltezar - Mechanical Systems and Signal Processing 18 (2004).

7. NASA Technical Memorandum 4793 - Wavelet Analyses of F/A-18 Aeroelastic and Aeroservoelastic Flight Test Data Martin J. Brenner - April 1997.

8. Non-Stationary Dynamics Data Analysis with Wavelet-SVD Filtering - M.J. Brenner - Mechanical Systems and Signal Processing 17 (2003) 765-786.

9. Time-Frequency Analysis for Transfer Function Estimation and Application to Flutter Clearance - Feron E., M. Brennen, J. Paduano and A. Turevski. Journal of Guidance, Control and Dynamics, 1988.

10. Aeroelastic Flight Data Analysis with the Hilbert-Huang Algorithm - Martin Brenner \& Chad Prazenica - NASA/TM-2006213665, January 2006.

11. Bivariate EMD-Based Data Adaptive Approach to the Analysis of Climate Variability - Khademul Islam Molla, Poly Rani Ghosh and Keikichi Hirose, Discrete Dynamics in Nature and Society, Volume 2011.

12. An Eigensystem Realization Algorithm (ERA) for Modal Parameter Identification and Model Reduction - Juang and Pappa, Proc. Of the Workshop on Identification and Control of Flexible Space Structures, Vol. 31985.

13. A Covariance Based Realization Algorithm for the Identification of Aeroelastic Dynamics from In-Fligth Data - Brennen and others - Proc. AIAA Atmospheric Flight Mechanics Conference, 2011.

14. Adaptive prediction and predictive control. London: Peter Peregrinus Ltd, 1995.

10

Journal of Aerospace Engineering

http://mc.manuscriptcentral.com/(site) 